\title{
Using experimental data to constrain theories of hopping conduction in NTD germanium
}

\author{
A. L. Woodcraft ${ }^{\mathrm{a}, 1}$, R. V. Sudiwala ${ }^{\mathrm{a}}$, E. Wakui $^{\mathrm{b}}, \mathrm{M}$ Piat $^{\mathrm{c}}$ \\ a Department of Physics and Astronomy, University of Wales, Cardiff, P.O. Box 913, Cardiff, CF24 $3 Y$ B, UK. \\ b Department of Physics, Queen Mary and Westfield College, Mile End Road, London, E1 4NS, UK \\ ${ }^{\mathrm{c}}$ Institut d'Astrophysique Spatiale, Bât. 121, Université Paris XI, F-91405 Orsay Cedex, France
}

\begin{abstract}
The mechanism for low temperature electrical conduction in neutron transmutation doped (NTD) germanium is believed to be variable range hopping $(\mathrm{VRH})$. The resistance, $R$, at temperature $T$ should then follow $R(T)=$ $R_{0} \exp \left(T_{0} / T\right)^{p}$, for constant (or nearly constant) values of $T_{0}, R_{0}$ and $p$. NTD Ge is thought to have a "Coulomb gap" in the density of states; theories then generally predict $p=0.5$. However, some theories suggest larger values, such as $p=0.55$. So far, experimental results have failed to distinguish between these values of $p$. We show that it is nevertheless practical to make sufficiently accurate measurements to do so. We present measurements for several NTD Ge samples with different doping levels, and discuss the various possible sources of error.
\end{abstract}

Keywords: variable range hopping; NTD Ge; Coulomb gap; semiconductor

The electronic transport mechanism in neutron transmutation doped (NTD) germanium [1] at low temperatures is believed to be variable range hopping (VRH), for which

$$
R(T)=R_{0} \exp \left(\frac{T_{0}}{T}\right)^{p}
$$

is predicted, where $R$ is the resistance at temperature $T$, and $T_{0}$ and $R_{0}$ depend on the doping and thermistor dimensions. At sufficiently low temperatures a Coulomb gap is expected to exist in the density of states. In this case, it is generally believed that $p=0.5[2]$. However, higher values of $p$

\footnotetext{
1 Corresponding author. E-mail:

A.Woodcraft@physics.org
}

Physica B, 329-333:1300-1301, 2003 have been predicted both numerically and analytically.

Experimental results are therefore needed in order to determine the correct value for $p$. Power laws are hard to determine experimentally, and available measurements do not provide good evidence for any of the suggested values of $p$. In this paper we show that it is nevertheless possible to make sufficiently accurate measurements to do so.

The measurements were made using a paramagnetic salt adiabatic demagnetisation refrigerator [3]. The main magnet is compensated to reduce the field at the sample to a level which should not affect the measurements. Sample resistances were measured either using a commercial resistance bridge or by voltage measurements using a 


\begin{tabular}{ccccc} 
Name & $T_{0}$ & $p$ & Doping density Minimum temp. \\
\hline 12 & 10.24 & $0.5611 \pm 0.0004$ & $9.79 \times 10^{16}$ & $100 \mathrm{mK}$ \\
19 & 16.26 & $0.472 \pm 0.001$ & $8.09 \times 10^{16}$ & $100 \mathrm{mK}$ \\
F & 62.21 & $0.487 \pm 0.002$ & $3.65 \times 10^{16}$ & $300 \mathrm{mK}$ \\
\hline
\end{tabular}

Table 1
Sample properties. As is customary, $T_{0}$ is quoted for fits fixing $p=0.5$. The minimum temperature is the lowest temperature the thermistor is designed to be used at.

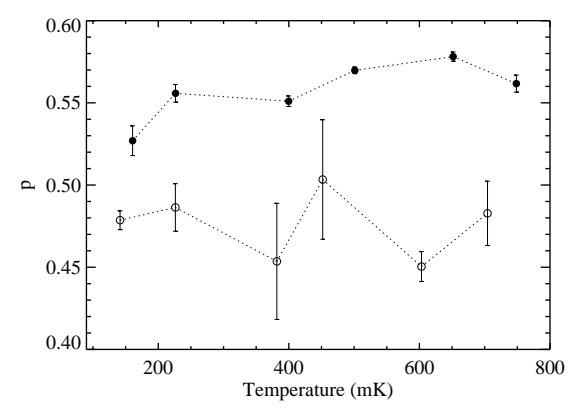

Fig. 1. Fitted values of $p$ over sub-sets of the full temperature range for samples $12(\bullet)$ and $19(\circ)$.

differential amplifier. In the latter case, the voltage was measured as a function of current [3]. Absolute thermometry was provided by a germanium secondary standard thermometer.

Measured values of $p$ (determined by fitting equation 1 to the data) are shown in Table 1 for three samples.

We have considered and rejected various possible sources of error. The values do not appear to be the result of random errors since they are repeatable, and similar NTD types produce similar values for $p$. The use of two different read-out systems for measurements suggests the results are not an artefact of the read-out system (such as a non-linear response). Moreover, comparison of two samples measured simultaneously with the resistance bridge showed results incompatible with both samples having the same value for $p$. The samples had similar resistances (and the bridge was on the same resistance range); systematic errors in the bridge are thus unlikely to be the cause.

The measured values of $p$ are not altered appreciably by omitting points at either end of the temperature range when fitting. In addition, sim-

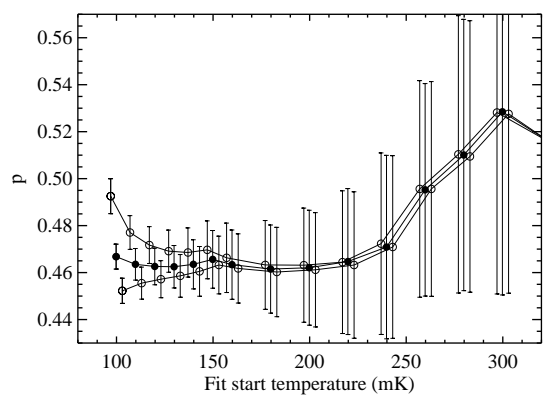

Fig. 2. Fitted values of $p$ for sample 19, varying the lowest temperature in the fitting range. The different datasets are offset slightly along the $x$ axis for clarity. Values used for the amplifier gain: correct $(\bullet), \pm 30 \%$ (०).

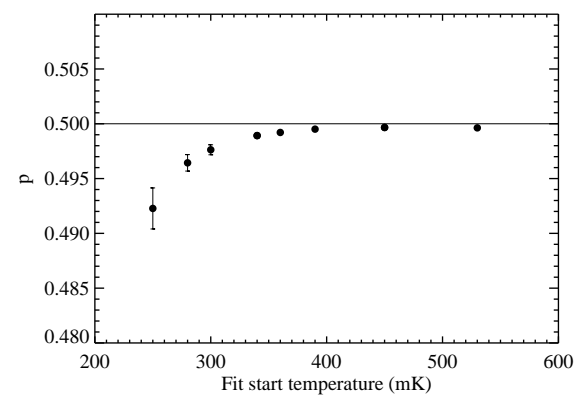

Fig. 3. Fitted values of $p$ using the same procedures as for the real data, for simulated noise-less data representing a thermistor with similar properties to sample F, but with $p=0.5$. The lowest temperature in the range fitted over is varied as in Fig. 2. Note that this thermometer is designed for operation at temperatures above $300 \mathrm{mK}$.

ilar values of $p$ are obtained when fitting to subsets of the data (Fig. 1). This suggests that we are measuring a true power law. A possible systematic error would appear if we used an incorrect value for the amplifier gain when analysing voltage measurements. However, Fig. 2 shows that a large error in gain is required to alter the results significantly, and then only for fits which include the lowest temperature points.

In equation $1, R$ is the resistance at zero bias. Due to self-heating and electric field effects [4], the thermistor resistance will alter with bias current; we obtain $R$ from voltage measurements by extrapolation of the low current data. Results from a simulation [5] (Fig. 3) show that differences be- 
tween the measured and actual zero bias resistance should not cause a significant error in $p$.

In conclusion, we have shown that it is possible to determine $p$ experimentally to sufficient accuracy to distinguish between different predicted values. We have considered and rejected various possible sources of error. The measured values appear to show a value which is different for different doping densities. Lack of space precludes a discussion of the theoretical implications ${ }^{2}$, other than to point out that the results are not in agreement with the standard prediction of $p=0.5$.

\section{Acknowledgement}

We thank J. Beeman, J. J. Bock and P. Mauskopf for supplying the samples measured.

\section{References}

[1] E. E. Haller, Infrared Phys. 25 (1985) 257-266.

[2] A. L. Efros, B. I. Shklovskii, J. Phys. C 8 (1975) L49-51.

[3] A. L. Woodcraft et al., International Journal of Infrared and Millimeter Waves 23 (2002) 575-595.

[4] J. Zhang et al., Phys Rev B 57 (1998) 4472-4481.

[5] M. Piat et al., Journal of Low Temperature Physics 125 (2001) 189-203.

2 Comparison with theory is complicated by the possibility of temperature variation of $R_{0}$. 